The Astrophysical Journal, 180:L11-L13, 1973 February 15

(c) 1973. The American Astronomical Society. All rights reserved. Printed in U.S.A.

\title{
DIFFUSE NEBULAE AT HIGH GALACTIC LATITUDES
}

\author{
G. Grasdalen* and J. G. Cohen \\ Department of Astronomy, University of California at Berkeley \\ Received 1972 September 11; revised 1972 November 24
}

\begin{abstract}
The nature of the diffuse nebulae at high galactic latitudes is discussed. Although these nebulae show $\mathrm{H} \beta$ in emission, evidence is presented that they are reflection nebulae illuminated by the integrated light of the Galaxy. Observational evidence for this suggestion is obtained by narrow-band photometry of external spiral galaxies with large entrance apertures. In the integrated spectra of spiral disks, we find that $\mathrm{H} a$ is in emission.

Subject headings: galaxies - nebulae
\end{abstract}

We present theoretical and observational evidence that the galactic nebulae at $|b|>20^{\circ}$ in which $\mathrm{H} \beta$ emission was observed by Johnson (1972) are reflection nebulae, rather than ionized regions.

These nebulae are the faintest catalogued by Lynds (1965), having in most cases brightnesses only barely detectable above the sky as seen in the Palomar Sky Survey. Arp (1965) has found that galaxies can be followed on these plates to a limit of 5 percent above the sky. For the discovery of extended sources, this implies that they are perhaps 10 percent above the sky in a band $1000 \AA$ wide centered on $4100 \AA$. (The transmission of the plate-filter combination for the Palomar Sky Survey is given by Minkowski and Abell 1963.) Emission in $\mathrm{H} \beta$ was found by Johnson (1972), who observed 49 nebulae with wide and narrow filters centered on $\mathrm{H} \beta$ and an aperture of 3.2 . His observations were converted to equivalent widths and corrected for the general absorption at $\mathrm{H} \beta$ seen in the night sky at high galactic latitudes (Johnson 1971). They imply a maximum equivalent width of emission at $\mathrm{H} \beta$ of $5 \AA$ and a mean value of $3 \AA$. Assuming an emission-line spectrum similar to that of the Orion Nebula (Johnson 1968), we must multiply this equivalent width by a factor of 4 to allow for the contribution of other emission lines within the bandpass of the blue plates. This computation implies a maximum enhancement in brightness over the sky of 2 percent in the blue Palomar Sky Survey, compared to a minimum detectable enhancement of 5 percent. If these nebulae are purely emission objects, we would not expect to see any of them on the blue Palomar Sky Survey plates. A similar calculation for the red Sky Survey plates shows that most of these nebulae would not be visible there either, if they are purely emission nebulae.

Since an additional source of light appears necessary, let us consider that these diffuse nebulae are entirely reflection nebulae, illuminated by the integrated light of the Galaxy. The concept of reflection nebulae illuminated in such a way was suggested previously by van den Bergh (1966).

If we take the surface brightness of the disk of our Galaxy to be that of an average of external spirals, the albedo of the reflecting material that is required (even for nebulae 0.5 times as bright as the sky) is less than 10 percent, so that the nebulae may have very small optical depths. This is consistent with the lack of obvious extinction in these objects. If we are to explain the observations of Johnson (1972) on the basis of reflected light, we must then show that in the integrated light from the disk of a spiral galaxy similar to our Galaxy $\mathrm{H} \beta$ is in emission.

* Present address: Kitt Peak National Observatory, Tucson, Arizona.

L11 
Let us assume that $W$ is the equivalent width of $\mathrm{H} \beta$ emission in the integrated spectrum of the disk of a spiral. If $\alpha$ is the ratio of brightness of the high-latitude reflection nebula to that of the sky at a wavelength near $\mathrm{H} \beta$, then the observed $\mathrm{H} \beta$ net equivalent width $X$ will be

$$
X=\frac{W \alpha}{1+\alpha} .
$$

For $X=3 \AA$, and $\alpha$ between 0.05 and 0.4 (a choice dictated by the fact that these nebulae can barely be seen on the Palomar Sky Survey), we require $11 \AA<W<65 \AA$.

Searle (1971) observed $\mathrm{H} \beta$ emission in the largest $\mathrm{H}$ II regions of external Sc galaxies which, when converted to an equivalent width, yields $150-200 \AA$ for the integrated spectrum of $\mathrm{H}$ II regions. If we estimate as 5 percent the fraction of the area of the disk which is covered by giant $\mathrm{H}$ II regions, and the relative brightness of the $\mathrm{H}$ II regions and the stars, we see that it may be possible that the emission from the $\mathrm{H}$ II regions overwhelms the stellar absorption, and that the integrated spectrum of a spiral disk will have $\mathrm{H} \beta$ in emission. This was the motivation for our observations of external spirals. Evidence for extensive ionized hydrogen in the spiral arms of our Galaxy is given by Davies, Matthews, and Pedlar (1972).

If emission is present in the integrated spectrum of a galaxy, we expect it to be stronger at $\mathrm{H} \alpha$ than at $\mathrm{H} \beta$ by a factor of 3 . Hence, we tried to detect $\mathrm{H} \alpha$ emission in four spiral galaxies with the Crossley 36-inch $(92-\mathrm{cm})$ telescope and a dual-channel scanner. The entrance aperture was $1^{\prime}(1.5 \mathrm{~mm})$, and the exit slit of the scanner was $32 \AA(1 \mathrm{~mm})$; however, due to the extremely large entrance aperture, the effective resolution is closer to $50 \AA$. The galaxies were observed at 5 wavelengths $100 \AA$ apart with 10-s integration times. This was repeated 10 times per galaxy. Because radial velocities are not known for some of the galaxies, the wavelengths at which we observed were fixed. The center of the aperture was at the nucleus, but in the case of NGC 628 a prominent knot in the arm east of the nucleus was also observed. In the other three cases, the galaxies are sufficiently small that the entrance aperture includes most of the galaxy. Guiding was difficult, and each scan probably covered a slightly different part of the galaxy. The results of our observations are given in table 1 . The source for the types, major diameters, visual magnitude, and radial velocities is de Vaucouleurs and de Vaucouleurs (1964). The equivalent widths in $\mathrm{H} \alpha$ are calculated assuming a $50 \AA$ bandpass. They should be accurate to \pm 50 percent for NGC 124 , and \pm 30 percent for the other galaxies. The apparent magnitude at $6500 \AA$ within a $1^{\prime}$ diaphragm $\left(V^{1}\right)$ should be accurate to $\pm 0.5 \mathrm{mag}$. A large part of this error is uncertainty in the exact value of the bandpass, due to our large entrance aperture. The particular galaxies

TABLE 1

Ha Emission of Spiral Galaxies

\begin{tabular}{|c|c|c|c|c|c|c|}
\hline Name & Type & Diameter $\left({ }^{\prime}\right)$ & $V$ & $v_{r}(\mathrm{~km}-1)$ & $W(\mathrm{H} a)(\AA)$ & $V^{*}$ \\
\hline NGC $124 \ldots \ldots \ldots$ & $\mathrm{Sc}-$ & 1.3 & & $\ldots$ & $31 *$ & 13.5 \\
\hline $\begin{array}{ll}\text { NGC } & 245 \\
\text { NGC } & 628\end{array}$ & $\mathrm{Sc}$ & 1.4 & 12.9 & $\ldots$ & 67 & 13.0 \\
\hline $\begin{array}{l}\text { (Nucleus) } \quad \ldots \ldots \ldots \\
\text { NGC } 628\end{array}$ & $\mathrm{Sc}^{-}$ & 9.0 & 10.0 & 653 & 0 & 11.7 \\
\hline $\begin{array}{c}(\text { Arm }) \\
\text { NGC } 772 \ldots \ldots \ldots \ldots\end{array}$ & $\ddot{\mathrm{S}} \dot{b}-$ & 5.4 & $\ddot{11.4}$ & 2431 & $\begin{array}{r}18 \\
5\end{array}$ & $\begin{array}{l}12.3 \\
12.1\end{array}$ \\
\hline
\end{tabular}

* Emission in band centered at $6662 \AA$ rather than at $6562 \AA$. 
were picked because they are small spirals, relatively bright, and conveniently located in the sky.

Apart from Seyfert galaxies, one would not expect strong emission lines in the nuclei of spiral galaxies, yet emission was seen in every case. We interpret the observed emission at $\mathrm{H}_{\alpha}$ as due to $\mathrm{H}$ II regions in the outer parts of these spiral galaxies. Furthermore, the emission is almost in the range required by equation (1), namely, $11 \AA<W<65 \AA$ at $\mathrm{H} \beta$, or a minimum of about $35 \AA$ at $\mathrm{H} \alpha$. The redshift of NGC 772 is such that $\mathrm{H}_{\alpha}$ lies midway between the two bandpasses centered on $6562 \AA$ and $6662 \AA$; this explains the low level of emission observed for this galaxy. Note the difference between the nucleus and the arm of NGC 628 .

The observed equivalent widths are at the lower limit of the required strength of emission of $\mathrm{H}_{\alpha}$. Because our apertures were centered on the nucleus, which has a greater surface brightness than the outer parts of the galaxy, the observations listed in table 1 underestimate the emission equivalent width that will be seen by a highlatitude reflection nebula. Such nebulae will be much closer to the disk than to the nucleus, so that the nuclear light (which has little or no emission) will have a larger dilution factor. Furthermore, if the nebula is directly above a giant $\mathrm{H}$ II region in the disk, the $\mathrm{H} \beta$ emission in the reflected light will be considerably enhanced.

We have shown by our observations that the integrated light of spirals (even including the nucleus) has $\mathrm{H} \alpha$ in emission. Johnson (1971) observed a dependence of $\mathrm{H} \beta$ emission in the night sky outside known $\mathrm{H}$ iI regions on the cosecant of galactic latitude. His observations may be explained by this description of the large-scale radiation field in spirals. Notice also that since the integrated spectral type of a galaxy is fairly red, it may be possible to explain the red color indices of the high-latitude nebulae compared to the more typical reflection nebulae surrounding stars.

Minkowski, Silk, and Siluk (1972) present evidence for a spatial correlation between the high-velocity gas observed in the $21-\mathrm{cm}$ line and the high-latitude nebulae, which they believed to be emission nebulae. We have demonstrated that a consistent interpretation of these objects may be obtained on the hypothesis that they are reflection nebulae. This eliminates the problem of exciting the gas discussed by them. However, the source of energy required to keep this material above the plane is not clear. In this sense, the correlation found by Minkowski et al. (1972) between the high velocity gas and the diffuse nebulae at high galactic latitude may be noncoincidental.

J.G.C. is grateful to the Miller Institute for Basic Research for financial support.

\section{REFERENCES}

Arp, H. C. 1965, Ap. J., 142, 402.

Bergh, S. van den. 1966, A.J., 71, 990.

Davies, R. D., Matthews, H. E., and Pedlar, A. 1972, Nature Physical Science 238, 101.

Johnson, H. M. 1968, Nebulae and Interstellar Matter, ed. B. Middlehurst and L. H. Aller (Chicago: University of Chicago Press).

- $1971, A$. A. J., 164, 379.

- 1972, ibid., 174, 591.

Lynds, B. T. 1965, Ap. J. Suppl., 12, 163.

Minkowski, R. L., and Abell, G. 1963, Basic Astronomical Data, ed. K. Aa. Strand (Chicago: University of Chicago Press), p. 481.

Minkowski, R. L., Silk, J., and Siluk, R. S. 1972, Ap. J. (Letters), 175, L123.

Searle, L. 1971, Ap. J., 168, 327.

Vaucouleurs, G. de, and Vaucouleurs, A. de. 1964, Reference Catalogue of Bright Galaxies (Austin: University of Texas Press). 